Solenoid-free Plasma Start-up in NSTX using Transient CHI

R. Raman, T. Jarboe, B. Nelson, D. Mueller, V. A. Soukhanovskii

January 7, 2009

Nuclear Fusion 
This document was prepared as an account of work sponsored by an agency of the United States government. Neither the United States government nor Lawrence Livermore National Security, LLC, nor any of their employees makes any warranty, expressed or implied, or assumes any legal liability or responsibility for the accuracy, completeness, or usefulness of any information, apparatus, product, or process disclosed, or represents that its use would not infringe privately owned rights. Reference herein to any specific commercial product, process, or service by trade name, trademark, manufacturer, or otherwise does not necessarily constitute or imply its endorsement, recommendation, or favoring by the United States government or Lawrence Livermore National Security, LLC. The views and opinions of authors expressed herein do not necessarily state or reflect those of the United States government or Lawrence Livermore National Security, LLC, and shall not be used for advertising or product endorsement purposes. 


\title{
Solenoid-free Plasma Start-up in NSTX using Transient CHI
}

R. Raman ${ }^{1}$, T.R. Jarboe ${ }^{1}$, D. Mueller ${ }^{2}$, B.A. Nelson ${ }^{1}$, M.G. Bell ${ }^{2}$, R. Bell ${ }^{2}$, D. Gates ${ }^{2}$, S. Gerhardt $^{2}$, J. Hosea ${ }^{2}$, R. Kaita ${ }^{2}$, H. Kugel ${ }^{2}$, B. LeBlanc ${ }^{2}$, R. Maingi ${ }^{3}$, R. Maqueda ${ }^{4}$, J. Menard ${ }^{2}$, M. Nagata 5 , M. Ono ${ }^{2}$, S. Paul ${ }^{2}$, L. Roquemore ${ }^{2}$, S. Sabbagh6 , V. Soukhanovskii ${ }^{7}, \mathrm{G}$. Taylor ${ }^{2}$

1) University of Washington, Seattle, WA, USA

2) Princeton Plasma Physics Laboratory, Princeton, NJ, USA

3) Oak Ridge National Laboratory, Oak Ridge, TN, USA

4) Nova Photonics, Princeton, NJ, USA

5) University of Hyogo, Himeji, Japan

6) Columbia University, New York, NY, USA

7) Lawrence Livermore National Laboratory, Livermore, NJ, USA

Email address of main author: raman@aa.washington.edu

\begin{abstract}
Experiments in NSTX have now demonstrated the coupling of toroidal plasmas produced by the technique of Coaxial Helicity Injection (CHI) to inductive sustainment and ramp-up of the toroidal plasma current. In these discharges, the central Ohmic transformer was used to apply an inductive loop voltage to discharges with a toroidal current of about $100 \mathrm{kA}$ created by $\mathrm{CHI}$. The coupled discharges have ramped up to $>700 \mathrm{kA}$ and transitioned into an H-mode demonstrating compatibility of this startup method with conventional operation. The electron temperature in the coupled discharges reached over $800 \mathrm{eV}$ and the resulting plasma had low inductance, which is preferred for long-pulse high performance discharges. These results from NSTX in combination with the previously obtained record $160 \mathrm{kA}$ non-inductively-generated startup currents in an ST or tokamak in NSTX demonstrate that CHI is a viable solenoid-free plasma startup method for future STs and tokamaks.
\end{abstract}




\section{Introduction}

The favorable properties of the Spherical Torus (ST) confinement concept arise from its small aspect ratio [1]. Reactors based on the ST are projected to operate at very high values of the toroidal beta (>40\%) and bootstrap current fraction (>90\%) [2]. The capability of the ST to operate at high beta and high values of the bootstrap current fraction has been demonstrated in NSTX [3]. However, elimination of the central solenoid is essential for the viability of the ST concept, making solenoid-free plasma start-up and non-inductive sustainment necessary for an ST based reactor or a component test facility. Solenoid-free plasma startup is also relevant to steady-state tokamak operation, as the central transformer coils of a conventional aspect-ratio tokamak reactor would be located in a high radiation environment but would be needed only during the initial discharge initiation and current ramp-up phases. Removing the central solenoid provides greater flexibility in the selection of the aspect ratio and simplifies the reactor design.

Coaxial Helicity Injection (CHI) is a promising candidate both for plasma startup and for edge current drive during the sustained phase. The possibility of using $\mathrm{CHI}$ in an ST was first proposed in the late 1980's [4]. At that time, it was generally believed that the development of non-axisymmetric MHD perturbations of the plasma equilibrium was needed for plasma startup using the CHI process in STs. This approach was initially investigated in NSTX and in several other STs. However, in a significant development during the past few years, it was shown that for the purpose of plasma startup, axisymmetric reconnection can produce a high quality startup equilibrium. This new method, referred to as transient $\mathrm{CHI}$, was first demonstrated on the HIT-II experiment at the University of Washington [5]. The method has now been successfully used on NSTX for a demonstration of solenoid-free plasma start up and successful coupling to subsequent inductive drive. The coupled discharges have ramped up to $>700 \mathrm{kA}$. In addition, discharges that used $4 \mathrm{MW}$ of neutral beam heating, transitioned 
into an H-mode demonstrating compatibility of this startup method with conventional highperformance H-mode operation.

\section{Transient CHI Startup in NSTX}

NSTX has a major/minor radius of $0.86 / 0.68 \mathrm{~m}$ and a toroidal magnetic field at the nominal major radius up to $0.55 \mathrm{~T}$. It is equipped with a central solenoid providing up to $0.7 \mathrm{~Wb}$ of inductive flux (double swung) which can generate plasma currents up to 1.5 MA. The outer poloidal field coils needed for equilibrium control are located about $0.5 \mathrm{~m}$ away from the plasma boundary. The entire plasma facing boundary of NSTX is composed of graphite tiles. NSTX routinely applies conventional helium and deuterium glow discharge cleaning as wall conditioning techniques. In the past, boronization of the plasma facing surfaces has been used but in the last two years, lithium coating of the lower divertor plates has been investigated and developed as an alternate wall conditioning method.

As shown in Figure 1, CHI is implemented by injecting current through the plasma from an external circuit along poloidal field lines that connect the lower divertor plates in the presence of a toroidal magnetic field. NSTX uses the lower divertor plates as the injector. The opposite end consisting of the upper divertor plates is referred to as the absorber. The ExB plasma drift is away from the injector region and into the absorber region. The initial poloidal field connecting the inner and outer divertor plates in the injector region is produced using the lower divertor coils, as shown in Figure 1.

For successful application of the transient CHI process, five requirements need to be satisfied. The first requirement is that there must be sufficient energy in the capacitor bank to produce the "bubble-burst" current, i.e. the injector current $I_{i n j}$ at which the $I_{\text {pol }} \times B_{T}$ force can overcome the field line tension in the injector, and cause the plasma to expand into the chamber above. The minimum injector current to meet the bubble burst condition is given as: 
$I_{i n j}=2 \psi_{i n j}^{2} /\left(\mu_{0}^{2} d^{2} I_{T F}\right)$, where $\psi_{i n j}$ is the poloidal flux at the injector insulating gap, $I_{T F}$ is the total current in the toroidal field coil and $d$ is the width of injector flux "footprint" [4] on the electrodes. For NSTX conditions, injector currents as low as $1.5 \mathrm{kA}$ satisfy this condition.

The second requirement is related to how quickly the CHI discharge can fill the vessel. This is dependent on the applied injector voltage as this sets the rate at which toroidal flux crosses the injector and absorber gaps: $V_{i n j}=d \phi_{t o r} / d t$. For nominal NSTX conditions and $1 \mathrm{kV}$ across the injector electrodes, the time needed to displace all of the toroidal flux within the vacuum vessel is about $1.4 \mathrm{~ms}$.

The third requirement is that there should be sufficient electrical energy in the capacitor bank to fully ionize and heat all of the injected gas. Typically, for deuterium gas, about $120 \mathrm{eV}$ per ion is needed for ionization and to increase the plasma temperature to $20 \mathrm{eV}$. This is generally a more difficult requirement to satisfy, as gas breakdown is easier at higher pressures where more energy is then required. On NSTX it has been possible to decrease the total amount of injected deuterium to $\sim 2$ Torr-liter, which is similar to the amount of gas normally used for inductive startup. Substantial reduction in the amount of gas needed for breakdown was realized by injecting it in a cavity below the gap between the lower divertor plates. Initial experiments at modest voltages, $\sim 1 \mathrm{kV}$, on the capacitor bank benefited from a $10 \mathrm{~kW}, 18 \mathrm{GHz}$ Electron Cyclotron Heating (ECH) pre-ionization source. However, at capacitor bank charging voltages of $1.5 \mathrm{kV}$ and higher, reliable gas breakdown was possible without the use of ECH. During 2008 ECH pre-ionization was not used in any of the CHI started discharges.

The fourth requirement relates the maximum final toroidal plasma current $I_{p}$ that can be produced to the energy available from the capacitor bank: $0.5 L_{p} I_{p}^{2}<E_{\text {cap }}=0.5 C V^{2}$. The inductance of the toroidal plasma current on typical equilibrium in NSTX is about $0.5 \mu \mathrm{H}$. 
For the present NSTX capacitor bank, the upper limit on the CHI produced toroidal plasma current is about $500 \mathrm{kA}$. The final requirement is that the flux footprints on the $\mathrm{CHI}$ electrodes should be sufficiently narrow. On NSTX the lower divertor coils located outside the vessel are used to provide the injector flux shaping.

For transient $\mathrm{CHI}$ discharge initiation, the toroidal field coils and the poloidal field coils needed to produce the desired flux conditions in the injector region are first energized. A preprogrammed amount of deuterium gas is then injected into the cavity below the gap between the inner and outer lower divertor plates. The $5-45 \mathrm{mF}$ capacitor bank charged at up to $1.7 \mathrm{kV}$ is connected by an ignitron switch to the lower divertor plates. The inner vessel and inner divertor plates act as the cathode, and the outer divertor plates and passive stabilizer plates act as the anode. This initiates the CHI discharge. After a programmed delay of 3 $10 \mathrm{~ms}$, when the plasma has expanded into the chamber and the toroidal plasma current is near its peak, the injector is short-circuited by an ignitron causing the injector current to rapidly decay. This produces reconnection of the magnetic field near the lower divertor plate region and causes the plasma column to detach from the injector region to form closed flux. An aspect of CHI plasma generation using this method is that flux closure can be demonstrated unambiguously by the persistence of plasma current after the injector current has been reduced to zero. During recent experiments in NSTX, induction was then applied to the closed-flux plasma equilibrium by ramping the current in the central solenoid to investigate coupling of the $\mathrm{CHI}$ produced current to conventional inductive operation.

\section{Experimental Results}

Figure 2 shows a discharge in which a $\mathrm{CHI}$ discharge is started with the central solenoid disconnected from its power supply. A $15 \mathrm{mF}$ capacitor charged to $1.7 \mathrm{kV}$ was used in this experiment to drive the injector current. In this discharge approximately $3 \mathrm{kA}$ of injector 
current produces nearly $120 \mathrm{kA}$ of plasma current, so the current multiplication, defined as the ratio of the plasma current to injector current, is about 40. The highest current multiplication obtained in NSTX CHI discharges is 70. At $10 \mathrm{~ms}$, with nearly zero injector current, about $60 \mathrm{kA}$ of toroidal plasma current remains on closed field lines. After the $\mathrm{CHI}$ injector current is reduced to zero, the $\mathrm{CHI}$ capacitor bank is no longer driving the plasma load. The only plausible explanation for the remaining toroidal plasma current is the creation of a closed flux equilibrium that then decays on an L/R time scale. The highest amount of closed-flux current produced in NSTX CHI discharges is $160 \mathrm{kA}$, which is a world record for non-inductively generated closed flux current in an ST or tokamak. In Reference 6, we show equilibrium reconstructions for $\mathrm{CHI}$ produced discharges based on fitting to external magnetic measurements, which demonstrate the presence of closed flux surfaces during the decay phase of the discharge.

In Figure 3a, we show traces for the injector current, the plasma current, and the applied inductive loop voltage for a CHI started discharge, similar to that shown in Figure 2 that was then coupled to induction. In this discharge approximately $3 \mathrm{kA}$ of injector current produces about $100 \mathrm{kA}$ of toroidal current initially. During the decay phase of this current, induction is applied from the central solenoid by ramping its current from zero with a rectifier power supply with an open-circuit voltage of $4 \mathrm{kV}$. The decrease in the applied loop voltage over time is due to resistance in the central solenoid circuit. The external poloidal field coil currents needed for equilibrium are pre-programmed during the first $40 \mathrm{~ms}$ of the discharge. After that the standard NSTX plasma control system [7] is used to control the plasma radial and vertical position, using real-time data from external flux loops and magnetic probes. This causes the decaying plasma current to ramp-up, reaching a peak value of $700 \mathrm{kA}$ in this case. In this discharge, starting at about $40 \mathrm{~ms}$, neutral beams are also injected at a power of $4 \mathrm{MW}$ to heat the plasma. During the NB heating, the discharge transitions into an H-mode at 160 
ms. Evidence of the H-mode is the characteristic drop in divertor $\mathrm{D}_{\alpha}$ emission (Figure 3a) and the development of a broad electron density $\left(\mathrm{N}_{\mathrm{e}}\right)$ profile measured by the Thomson scattering diagnostic (Figure $3 b$ ). Figure $3 b$ also shows the electron temperature $\left(T_{e}\right)$ from Thomson scattering and the ion temperature $\left(\mathrm{T}_{\mathrm{i}}\right)$ profile from charge-exchange recombination spectroscopy (CHERS) for the discharge after the transition into H-mode. At $180 \mathrm{~ms}$, the discharge has low internal inductance $l_{i}=0.64$, central safety factor $\mathrm{q}(0)=1.4$, elongation $\kappa=$ 2.1 and an average triangularity $\delta=0.47$. The fact that a $\mathrm{CHI}$ initiated discharge is able to transition to a discharge that has features suitable for a high-performance long-pulse operation bodes well for the application of this startup method to future machines.

In this discharge the loop voltage peaks about $0.9 \mathrm{~ms}$ after the time of peak plasma current. Although the timing of the loop voltage application has been varied during these coupling studies, a detailed scan of the timing of the loop voltage application with the peak in the plasma current has not yet been conducted in optimized discharges. In studies so far, further delaying the time of inductive ramp reduces the coupling to induction. Moving it earlier in time has not shown a dramatic improvement, but those scans were conducted in un-optimized discharges. After higher startup current optimized discharges are obtained, the timing of the loop voltage will be studied in more detail to reduce the drop in plasma current after the application of the inductive ramp.

The first attempts to add inductive drive to $\mathrm{CHI}$ initiated discharge resulted in no increase in the plasma current. However, in these discharges there was a significant increase in the O-II emission when the loop voltage was applied. It was not until extensive conditioning was performed in the form of $\mathrm{D}_{2}$ glow discharge cleaning (GDC) and electrode discharge conditioning that the plasma current was able to be increased by induction. Evidence for the need to condition the walls and/or divertor plates is shown in Figure 4. Shots taken with 1,2, 
and 3 capacitor banks $(5,10$ or $15 \mathrm{mF})$ in the $\mathrm{CHI}$ system differed primarily in the intensity of the low-Z (C and $\mathrm{O})$ impurity emission. The plasma current traces show that as the size of the capacitor bank is increased, the magnitude of the attained plasma current increases. Using 1 capacitor module $(5 \mathrm{mF})$, the maximum in the plasma current is only $40 \mathrm{kA}$, with 3 modules $(15 \mathrm{mF})$, the attained plasma current increases to $140 \mathrm{kA}$. However, as the magnitude of the plasma current increases, its rate of increase during the subsequent inductive phase actually decreases. The discharge that uses three capacitors has the fastest current decay rate immediately after the $\mathrm{CHI}$ pulse and does not couple well to induction, even though the input power is highest for this discharge. This behavior is understood by examining the filter scope signals that show the oxygen and carbon line radiation signals in the upper and lower divertor region. These signals increase as the magnitude of the injector current increases. At a higher level of injector current more of the electrode surface contaminants are liberated into the plasma and contribute to increased energy losses during startup. The discharge with three capacitors also had increased levels of absorber arc current, which occurs when part of the injector current bridges the upper divertor gap and flows along the upper insulator surface, thereby producing additional contaminants from the absorber region. In principle, as demonstrated on HIT-II [5], the absorber arc can be minimized or eliminated through control of the magnetic field pattern in the upper divertor region to minimize the amount of poloidal flux that connects the upper divertor plates.

As seen in Figure 4, with one and two capacitors, the line radiation signals do not change much, but with two capacitors there is a small increase in the signal from a wide-angle bolometer with a view over most of the plasma below the mid-plane of NSTX. Both the one and two capacitor cases couple well to induction, but the rate of current increase is higher for the one capacitor case. With three capacitors, however, the spectroscopic signals are significantly elevated and so is the radiated power signal. As demonstrated in experiments on 
HIT-II [5], CHI discharges cannot couple to induction if the radiated power approaches the input Ohmic power. For these plasmas with about $3-4 \mathrm{~V}$ of applied loop voltage and a plasma current of $100 \mathrm{kA}$, the input Ohmic power is less than $300-400 \mathrm{~kW}$ during the inductive coupling phase. Thus it is essential that during the startup phase, either (a) the radiated power be lower than the input Ohmic power, or (b) some form of auxiliary heating system such as ECH be used to heat the target plasma. During startup, because the plasma temperature is less than $20 \mathrm{eV}$, the dominant radiation comes from low- $\mathrm{Z}$ impurities such as carbon and oxygen. For the graphite divertor plates used in NSTX, it is essential that surface contaminants be significantly reduced. On HIT-II, which used graphite for the center stack and tungsten-coated stainless steel for the outer vessel, even with $30 \mathrm{kA}$ of injected current the radiated power could be kept lower than the input Ohmic power [5]. Because of this, HITII discharges initiated by $\mathrm{CHI}$ showed significant saving of the inductive flux required to reach a given plasma current. A corresponding saving of inductive flux has not yet been demonstrated in NSTX. For the near term, low-Z impurity radiation could be reduced on NSTX through an extensive high-current electrode discharge cleaning effort to remove impurities, notably water, from the graphite tiles in the injector. Additionally, newly installed coils in the upper divertor region will be used to eliminate the absorber arcs which also contribute impurities. Finally, a $350 \mathrm{~kW}$ ECH system capable of heating the startup plasma will be implemented on NSTX during 2010. With these techniques, NSTX discharges should be able to achieve reductions in the inductive flux consumption. It is useful to note that in HIT-II nearly the entire CHI started current is retained in the subsequent inductive ramp [5]. If the impurity levels in NSTX are sufficiently reduced, then approximately $34 \mathrm{mWb}$ of central solenoid flux would be saved for a startup current of $100 \mathrm{kA}$ and about $87 \mathrm{mWb}$ would be saved for a startup current of 300kA. In NSTX for zero pre-charge in the central solenoid $350 \mathrm{mWb}$ is available from the central solenoid for current ramp up. 
However, it is more important to note that for a reactor that has no central solenoid, the essential requirement for start-up is that solenoid-free started currents be capable of coupling to an alternate non-inductive current drive system, and this can be made easier using additional heating sources. Additionally, future machines are likely to use metal rather than carbon over a large part of the divertor plates to reduce tritium retention, and this may reduce radiation from low-Z impurities during plasma startup.

Recently the possible benefits for CHI discharges of lithium coating applied to the lower divertor plates were also tested. During these experiments, lithium was evaporated from two ovens mounted at the top of the vacuum vessel onto the lower divertor region at a rate of $10 \mathrm{mg} / \mathrm{minute}$ for 10 minutes prior to the initiation of a $\mathrm{CHI}$ discharge. The evaporator system is described in the 2008 IAEA Fusion Energy Conference proceedings [8]. The immediate benefit of lithium was to make the $\mathrm{CHI}$ started discharges more reproducible. This is probably because of the fact that lithium has a much lower ionization energy $(\sim 5 \mathrm{eV})$ than deuterium $(13 \mathrm{eV})$, and can act as a pre-ionizing source for the bulk of the injected deuterium. It is useful to note that an effort to initiate $\mathrm{CHI}$ discharges in helium (ionization energy $~ 25$ $\mathrm{eV}$ ) was much more difficult. The application of lithium reduces recycling of deuterium at the electrode surfaces, and it was anticipated that this would lower the density of the CHI produced plasma. At lower density the plasma should heat up to a higher temperature, which should reduce the $\mathrm{L} / \mathrm{R}$ decay rate and thereby increase the current at the time that induction was applied. A similar effect was seen on the HIT-II experiments when titanium gettering was employed as a wall conditioning technique. However, despite the benefit of lithium conditioning to the reproducibility of CHI startup in NSTX, the magnitude of the initial plasma current produced by $\mathrm{CHI}$ did not increase with the use lithium. The reason may be 
that the amounts of lithium used in present experiments were too small to observe the pumping effect on the fast time scale of the plasma initiation. However, on a longer time scale wall pumping was observed as shown in Figure 5, which compares two CHI started discharges with and without the application of lithium, but similar in other respects. After coupling to induction, the discharge with lithium has a much lower density and reaches a higher plasma current.

\section{Summary and Conclusions}

Using the method of transient CHI in NSTX, $160 \mathrm{kA}$ of closed-flux toroidal current has been produced. Now, for the first time in NSTX, CHI started discharges have been successfully coupled to induction to show compatibility between CHI started discharges and the conventional inductive approach. While results similar to this have been previously demonstrated on the smaller HIT-II experiment, this is the first such demonstration on a large ST with a poloidal field configuration more prototypical of a compact ST reactor such as the Component Test Facility (CTF). Another significant new result is achieving a current multiplication factor up to 70 , which is an order of magnitude larger than achieved in previous smaller experiments, and suggests a favorable scaling of the technique with machine size. The transitioning of the CHI-produced discharges in NSTX to a high confinement $\mathrm{H}-$ mode with low plasma inductance demonstrates the potential for the application of this method to future machines.

\section{Acknowledgments}

We acknowledge the support of the NSTX team for operation of the machine systems and diagnostics. Special thanks are due to E. Fredd, R. Hatcher, S. Ramakrishnan, C. Neumeyer for support with CHI related systems, and to Dr. N. Nishino of Hiroshima University for providing a fast camera that was used in some of the experiments. The fast camera images in 
this paper are from a fast camera provided by Nova Photonics, Inc. This work is supported by

US DOE contract numbers FG03-96ER5436, DE-FG03-99ER54519 and DE-AC02$76 \mathrm{CH} 03073$.

\section{References}

[1] KAYE, S.M., ONO, M., PENG, Y-K. M., et al., Fusion Technol. 36, 16 (1999)

[2] NAJMABADI, F. and the ARIES Team, Fusion Eng. Design, 41, 365 (1998)

[3] BELL, M.G., BELL, R.E., GATES, D.A., et al., Nuclear Fusion 46, S565-S572 (2006)

[4] JARBOE, T.R., Fusion Technol. 15, 7 (1989)

[5] RAMAN, R., JARBOE, T.R., HAMP, W.T., et al., Physics Plasmas 14, 022504 (2007)

[6] RAMAN, R., NELSON, B.A., BELL, M.G., JARBOE, T.R., MUELLER, D., et al., Physical Review Letters, 97, 175002 (2006).

[7] GATES, D.A, et al., Nuclear Fusion 46, 17 (2006)

[8] KAITA, R., et al., "Plasma performance improvement with Lithium coated plasma-facing components in NSTX," in Fusion Energy 2008 (Proc. $22^{\text {nd }}$ Int. Conf., Geneva, 2008) (Vienna: IAEA) CD-ROM file EX/P4-9 and http: //wwwnaweb.iaea.org/napc/physics/FEC/FEC2008/html/index.htm

This work performed under the auspices of the U.S. Department of Energy by Lawrence Livermore National Laboratory under Contract DE-AC52-07NA27344. 


\section{Figure Captions}

Fig.1: (a) On the left, is a line drawing showing the main components in NSTX required for plasma startup using the CHI method. Top-right (b) fast camera fish-eye image of the plasma during the early phase of plasma growth and (c) later in time after the CHI started discharge has filled the vessel.

Fig. 2: Injector and plasma currents for a discharge in which the central solenoid was disconnected from its power supply. Note that the magnitude of the plasma current is many times that of the injector current. After the injector current is reduced to near zero, the persisting plasma current indicates the presence of a decaying closed flux equilibrium.

Fig. 3: (a) Traces from a CHI started discharge that was coupled to induction using the central solenoid. Shown are the CHI injector current, the resulting CHI produced plasma current, the preprogrammed loop voltage, the divertor $\mathrm{D}_{\alpha}$ signal and the applied neutral beam power. (b) Shown are the electron temperature and density profiles from a Thomson scattering diagnostic soon after transition to $\mathrm{H}$-mode and the ion temperature profile from a CHERS diagnostic. Note the steepening in the edge density gradient during the H-mode phase.

Fig. 4: Left - Injector and Plasma current traces for discharges operated with 5, 10 and $15 \mathrm{mF}$ capacitor banks and the radiated power as measured by a wide angle bolometer. Right - shown are the resulting line radiation signals from oxygen and carbon in the injector and absorber regions. Shot 128400 used a single capacitor module $(5 \mathrm{mF}, 7.2 \mathrm{~kJ})$, shot 128401 used two capacitors ( $14.4 \mathrm{~kJ}$ initial stored energy in the capacitors) and shot 128402 used three capacitors (21 kJ).

Fig 5: Comparison of discharges with and without the application of a Lithium evaporative coating on the lower divertor plates in NSTX. In the discharge with Li conditioning, the plasma density significantly decreases over time. 
Figure 1
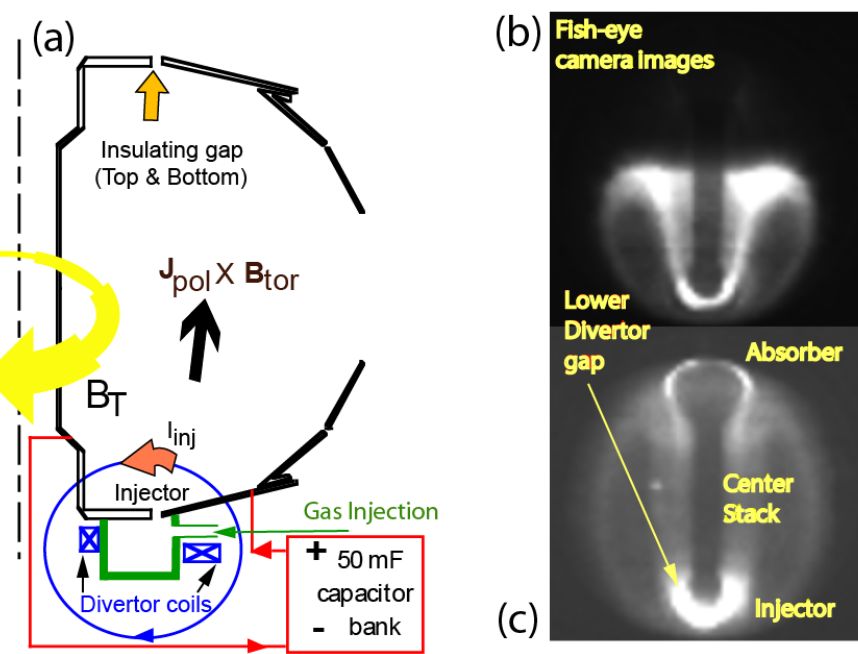

Figure 2

118346

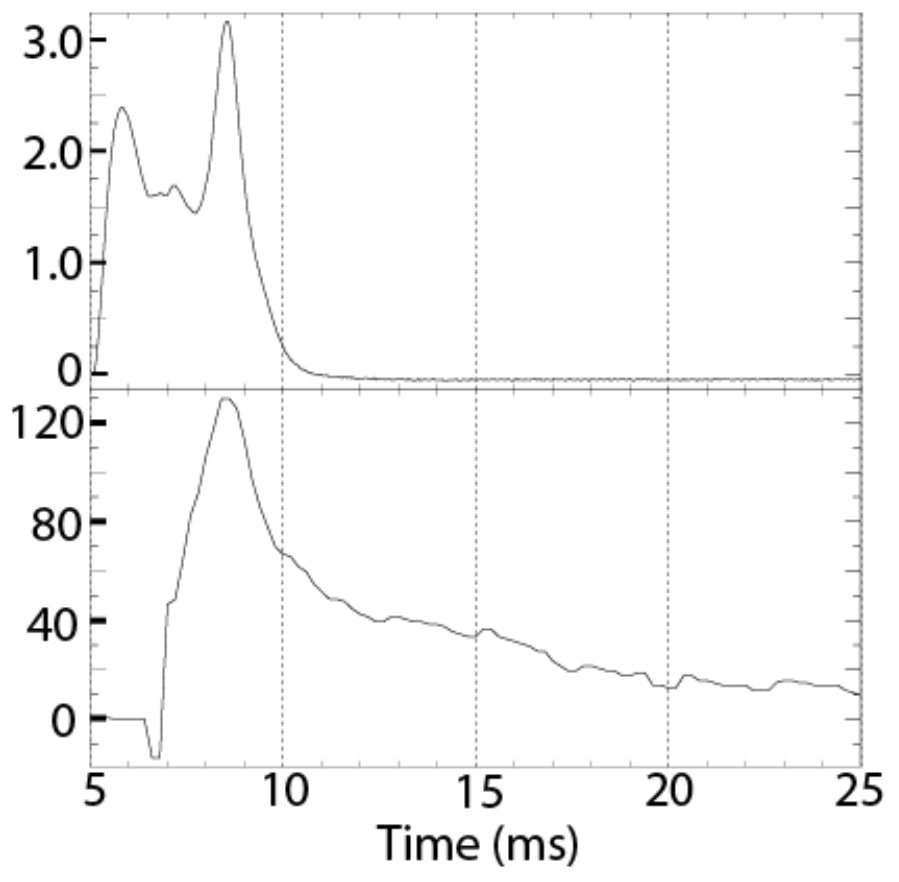


Figure 3
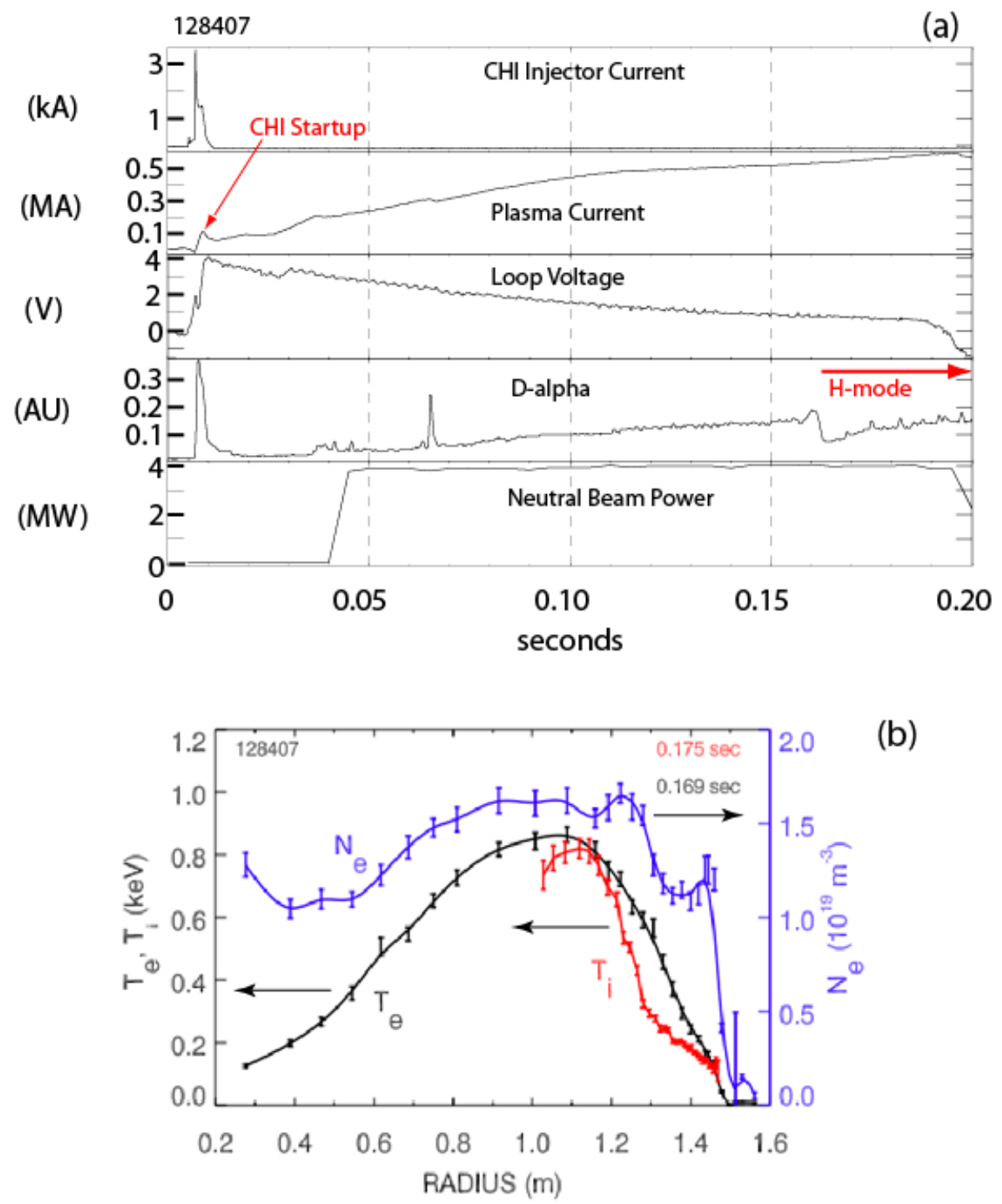

(b) 
Figure 4
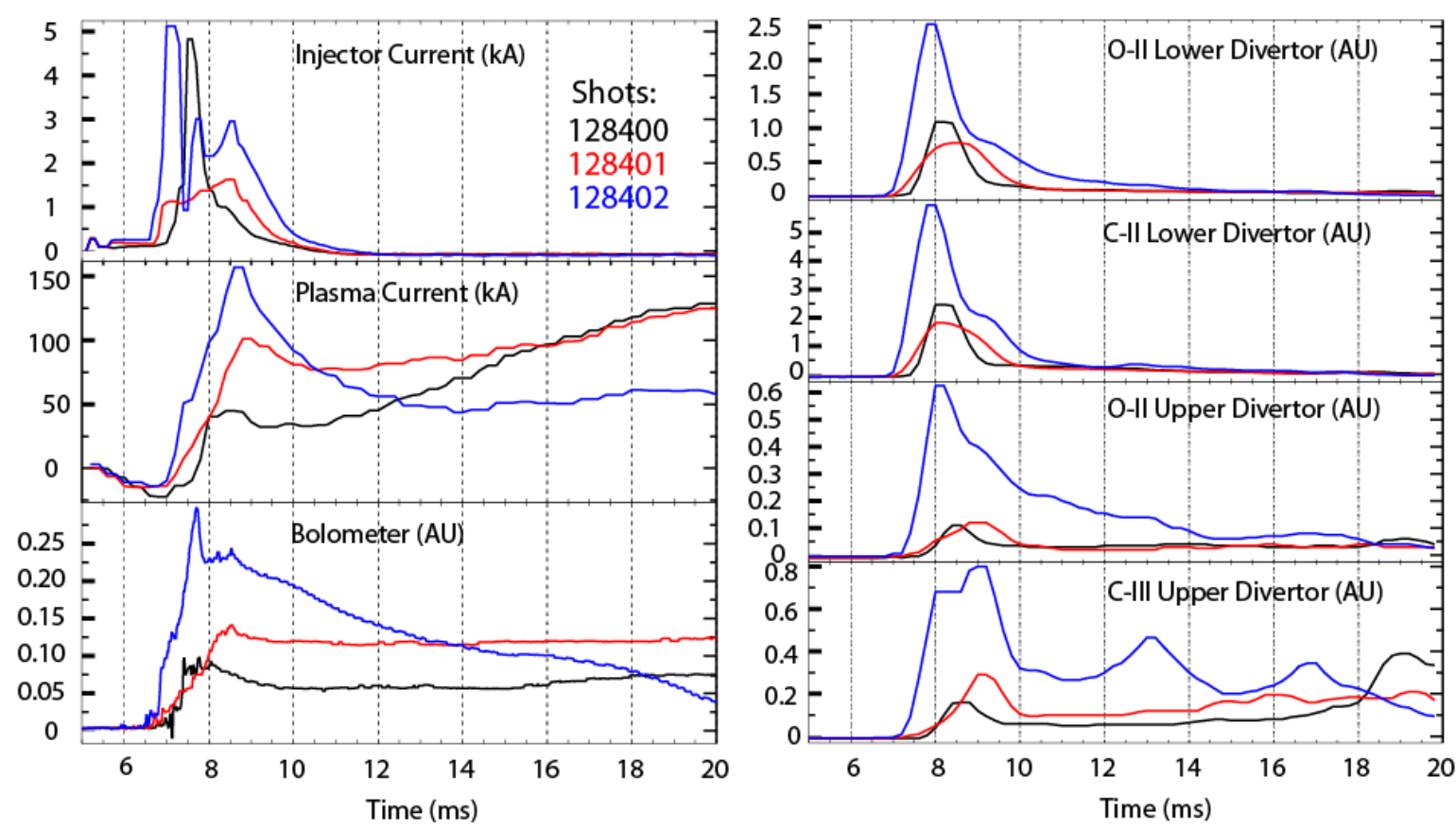

Figure 5

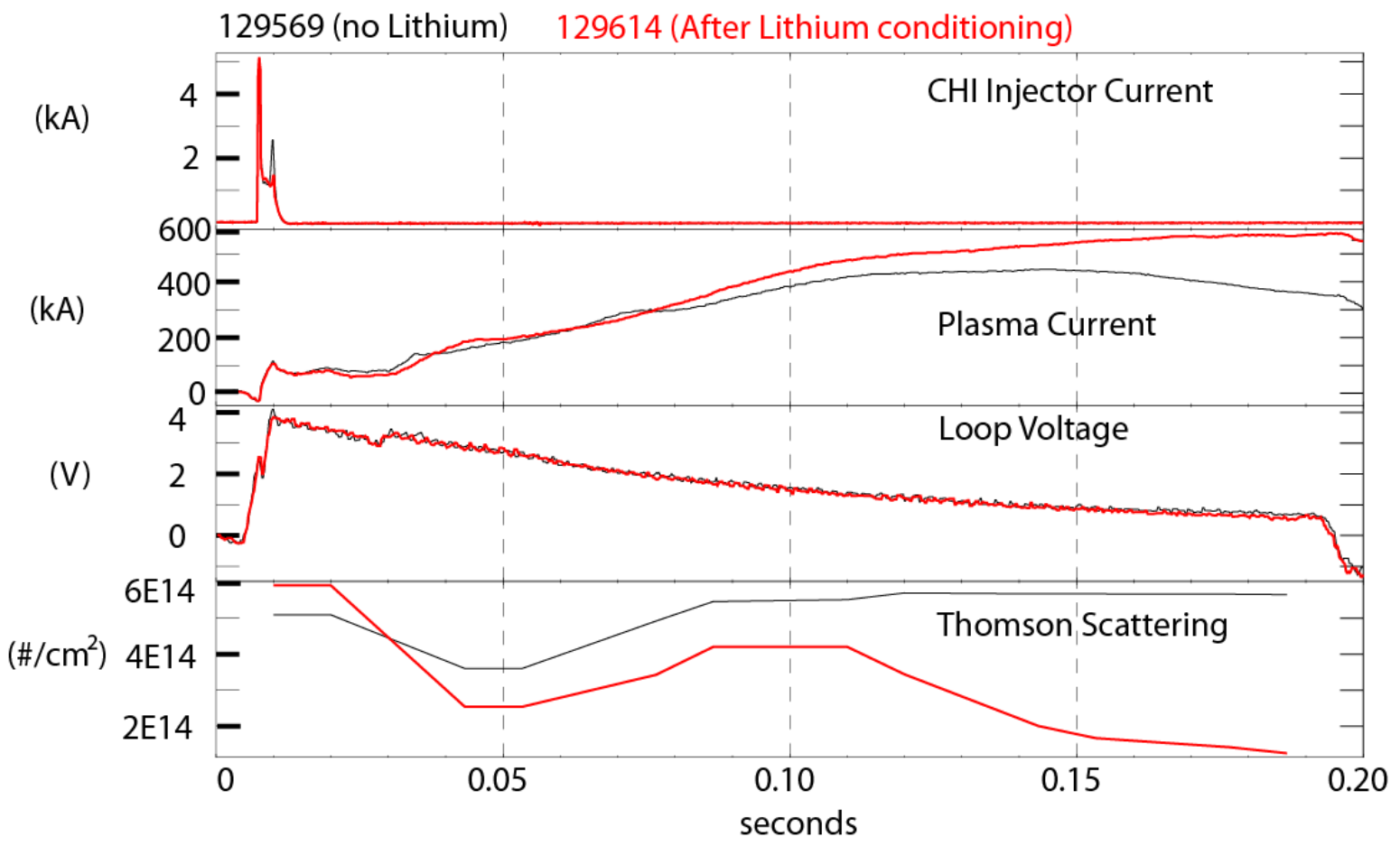

